\title{
Conseil juridique et communication scientifique
}

Francis André

Chargé de mission, DIST CNRS

Francis.Andre@cnrs-dir.fr

Renaud Fabre

Directeur, DIST CNRS

Renaud.Fabre@cnrs-dir.fr

Coline Ferrant

Doctorante, Sciences Po Paris / Northwestern University Evanston IL

coline.ferrant@sciencespo.fr

Joachim Schöpfel

Maître de conférences, Univ. Lille

joachim.schopfel@univ-lille3.fr

La recherche est en mutation profonde, tant dans sa façon de produire de la connaissance que dans les pratiques de communication de ses résultats. Développement rapide des technologies numériques de l'information et de la communication, d'infrastructures produisant des données massives, de moyens d'analyses et de simulation sans précédents, autant de facteurs qui structurent le mouvement vers une Science Ouverte. Ce mouvement impacte tous les acteurs de l'écosystème de la recherche : financeurs, éditeurs, organismes de recherche, chercheurs.

Les chercheurs, premiers maillons de la chaîne de la connaissance, sont les garants des bonnes pratiques de collecte et traitement de données puis de production et valorisation des résultats scientifiques. Dans ces activités, en pleine mutation dans le contexte numérique, ils sont confrontés à des questions juridiques non triviales.

\section{Une enquête dans les laboratoires du CNRS}

Dans le cadre de sa mission de définition de la stratégie IST du CNRS, la Direction de I'Information Scientifique et Technique (DIST) a réalisé une enquête sur les usages et besoins dans le domaine de I'information scientifique et technique auprès des directeurs des 1018 unités de recherche rattachées au CNRS, à la mi-2014. L'échantillon - 432 réponses (42\%) - est représentatif des 10 instituts disciplinaires du CNRS ${ }^{1}$. L'enquête portait sur la documentation, la publication et la valorisation de la recherche, notamment sur le libre accès à l'information (open access) et les données de la recherche. Quelques questions concernaient les problèmes et besoins juridiques liés à la pratique quotidienne de I'IST.

${ }^{1}$ Cf. http://www.cnrs.fr/fr/organisme/presentation.htm dont notamment I'Institut des sciences biologiques (INSB), I'Institut de chimie (INC), I'Institut des sciences humaines et sociales (INSHS) et Institut des sciences de l'ingénierie et des systèmes (INSIS). 


\section{Publications scientifiques}

Parmi les questions juridiques auxquelles ont été confrontés les directeurs d'unité ressortent celles liées aux publications scientifiques: I'utilisation ou la réutilisation des contenus auxquels l'unité a accès (mentionné par $38 \%$ de l'ensemble des répondants), la numérisation et la mise en ligne des fonds documentaires de l'unité (37\%), l'autorisation donnée à des tiers d'utiliser ou de réutiliser des contenus créés dans l'unité (35\%) et le dépôt de publications dans une archive ouverte alors qu'un contrat est conclu avec un éditeur (32\%).

Toutefois, ces questions concernent essentiellement les unités de sciences humaines et sociales (SHS) et, dans une moindre mesure, celles de sciences de la nature et de sciences formelles. Ainsi, par exemple, I'utilisation ou la réutilisation des contenus internes est mentionnée par $57 \%$ des directeurs d'unités de sciences humaines et sociales, contre $28 \%$ de celles de biologie et $15 \%$ de celles de mathématiques.

\section{Données de la recherche}

Un deuxième ensemble de questions juridiques auxquelles ont déjà été confrontés les directeurs d'unité a trait aux données de la recherche : la valorisation de données, notamment à caractère personnel (26\%) et l'ouverture de données, notamment issues de l'Open Data ou de sources publiques et administratives (23 $\%)$.

Ces questions concernent aussi davantage les unités SHS que celles de sciences de la nature et de sciences formelles. L'ouverture des données est ainsi mentionnée par $49 \%$ des directeurs d'unités SHS, contre 11 $\%$ de celles de biologie et $5 \%$ de celles de mathématiques. Cela va de pair avec une méconnaissance générale du statut juridique des données puisque $29 \%$ des directeurs d'unité pensent que les données brutes sont libres de droits, $39 \%$ qu'elles ne sont pas libres de droits, et $32 \%$ ne savent pas.

\section{Compétences et besoins}

D'une manière générale, le droit numérique de l'IST est jugé mal stabilisé et peu maîtrisé par les chercheurs et les professionnels de l'IST, surtout dans le domaine de la diffusion des documents et des données de la recherche en libre accès. Au niveau des directeurs, l'expérience rime souvent avec compétence mais aussi avec réalisme. Ceux qui ont déjà été confrontés à des questions juridiques se considèrent davantage compétents, mais expriment aussi davantage le besoin d'un conseil expert. Par exemple, $14 \%$ des directeurs d'unités SHS qualifient d'ignorance le niveau de compétence général de leur unité quant aux règles éthiques et juridiques, contre $22 \%$ de celles de biologie et $50 \%$ de celles de mathématiques. Parallèlement, $28 \%$ des directeurs d'unités SHS qualifient de grand l'intérêt d'un accompagnement juridique des unités, contre $9 \%$ de celles de biologie et $0 \%$ de celles de mathématiques.

\section{Réponses institutionnelles}

Au sein du CNRS, la direction des affaires juridiques (DAJ) assure un rôle de conseil, d'expertise, de veille juridique et de défense des intérêts de l'établissement ${ }^{2}$. Son offre de conseil et assistance juridique inclut les NTIC, le droit d'auteur et la propriété industrielle mais s'adresse surtout au directoire, aux directions fonctionnelles et administratives, aux instituts et délégations régionales, mais non aux chercheurs euxmêmes. Cependant, la DAJ anime également un réseau d'environ 80 juristes " e-loi " proche du terrain dont l'objectif est d'identifier et de reconnaître une véritable fonction juridique (en dehors des ressources

\footnotetext{
${ }^{2}$ http://www.dgdr.cnrs.fr/daj/orga/missions.htm
} 
humaines), bien répartie et homogène au sein du CNRS en faisant jouer les complémentarités entre les fonctions d'expertise de la DAJ et la mise en œuvre en régions.

À ceci, on ajoutera deux autres instances. (1) L'équipe du correspondant informatique et liberté (CIL) ${ }^{3}$ qui forme, informe et conseille pour toutes les questions relatives à la protection des données personnelles et qui anime son réseau de correspondants IL auprès des instituts scientifiques et délégations régionales. (2) Le Comité d'éthique du CNRS (COMETS ${ }^{4}$ ), instance consultative indépendante, placée auprès du Conseil d'administration du CNRS. Le COMETS traite des questions dont le saisissent le Conseil d'administration, le Conseil scientifique ou le Directeur général du CNRS, a la faculté d'autosaisine mais ne traite pas directement des cas individuels. Parmi les avis récents du COMETS, on citera l'intégrité scientifique, les publications scientifiques dans les réseaux sociaux et les medias, les sciences citoyennes, et les enjeux éthiques du partage des données scientifiques ${ }^{5}$.

Sur le terrain, les professionnels de l'information contribuent à cette fonction de conseil et assistance au plus près des chercheurs. Une enquête récente ${ }^{6}$ révèle que plus de la moitié des documentalistes font connaître à leurs usagers les évolutions du droit de l'information liées aux nouveaux modèles éditoriaux et de publication. Ils publient les informations sur le site du laboratoire ou de la documentation, organisent des ateliers pratiques et/ou diffusent une lettre d'information, sans négliger les moyens plus informels, les discussions à la cafétéria ou le conseil individuel. Cet investissement porte sur le droit d'auteur mais aussi sur les contrats d'éditeurs et l'accès aux ressources et, dans une moindre mesure, sur la protection des données personnelles et le droit de prêt.

\section{Perspectives}

Le projet de loi pour une République numérique et la nouvelle politique européenne en faveur d'une science ouverte font bouger les lignes. Les productions de recherche méritent un cadre juridique stabilisé, tant pour les publications (qui prennent des formes nouvelles avec les développements des collaborations massives) et pour les données (dont la valorisation est en pleine croissance). L'offre de service doit s'adapter.

Ailleurs, dans d'autres organismes scientifiques, le conseil juridique des chercheurs est tantôt à la charge de la bibliothèque ou du service responsable des données ou des publications, tantôt proposé par un service ou une équipe de juristes au sein d'une direction des services généraux. Dans la mesure où le conseil juridique demande une certaine expertise et expérience, il est souvent proposé d'une façon centralisée, au plus haut niveau d'un institut ou d'un organisme. C'est le cas du CSIRO (Australie) où un conseil juridique pour les auteurs est assuré par une équipe dédiée (legal team), en dehors de la bibliothèque. À la Max-Planck Gesellschaft (Allemagne), le service de la Digital Library (MPDL) répond aux demandes d'aide d'ordre juridique (contrats, litiges), malgré des moyens limités; leur service d'aide à la publication se situe entre facilitation et médiation - aider à faire et surtout, apprendre à faire. Une partie de l'assistance juridique passe par l'information sur le Web, via des sites d'information, des foires aux questions etc. Trois domaines surtout sont concernés: les contrats d'éditeur, le droit de déposer dans une archive ouverte, et le partage des données.

\footnotetext{
${ }^{3}$ http://www.cil.cnrs.fr/CIL/

${ }^{4}$ http://www.cnrs.fr/comets/

${ }^{5}$ http://www.cnrs.fr/comets/spip.php?article3

${ }^{6}$ DIST, 2015. Enquête personnel IST. CNRS, Paris. http://www.cnrs.fr/dist/Documents.html
} 
Mais même dans ce domaine très pointu, la Max-Planck Gesellschaft souhaite passer d'une offre de conseil centralisé (cellule juridique au sein de la MPDL) à un fonctionnement en réseau, composé d'experts en droit d'auteur de contrat d'éditeur (pas nécessairement des juristes) au niveau des instituts scientifiques. Ceci en raison du manque de disponibilité de la petite équipe centrale et de la nécessité d'un service capable de répondre aux questions des chercheurs en temps réel. Une question d'efficacité en d'autres termes, mettant en priorité un service efficace aux chercheurs afin de les aider à publier dans les meilleures conditions.

Suite aux enquêtes sur le terrain, le CNRS envisage un "schéma directeur juridique " relatif au droit numérique de l'IST pour répondre aux besoins de clarification en la matière, définir et organiser un service d'appui ad hoc. Pour les professionnels de l'information, cela passera également par un plan de formation national pour l'évolution de leurs compétences et impliquera les réseaux métiers (RENATIS, MEDICl, $\left.\operatorname{RBDD}^{7}\right)$.

\footnotetext{
${ }^{7}$ Réseau national de l'information scientifique et technique http://renatis.cnrs.fr/ - Réseau des métiers de l'édition scientifique publique (Médici) http://medici.in2p3.fr/ - Réseau Base de données http://rbdd.cnrs.fr/
} 